\title{
AN EXTENSION OF THE FLOATER-HORMANN FAMILY OF BARYCENTRIC RATIONAL INTERPOLANTS
}

\begin{abstract}
GEORGES KLEIN
Dedicated to Professor Jean-Paul Berrut on the occasion of his sixtieth birthday.

AbStRact. The barycentric rational interpolants introduced by Floater and Hormann in 2007 are "blends" of polynomial interpolants of fixed degree $d$. In some cases these rational functions achieve approximation of much higher quality than the classical polynomial interpolants, which, e.g., are ill-conditioned and lead to Runge's phenomenon if the interpolation nodes are equispaced. For such nodes, however, the condition of Floater-Hormann interpolation deteriorates exponentially with increasing $d$. In this paper, an extension of the Floater-Hormann family with improved condition at equispaced nodes is presented and investigated. The efficiency of its applications such as the approximation of derivatives, integrals and antiderivatives of functions is compared to the corresponding results recently obtained with the original family of rational interpolants.
\end{abstract}

\section{INTRODUCTION}

In 2007, Floater and Hormann introduced a family of barycentric rational interpolants [12]. For the interpolation of a function $f:[a, b] \rightarrow \mathbb{R}$ whose values are given at $n+1$ distinct nodes $a=x_{0}<x_{1}<\ldots<x_{n}=b$, they choose a nonnegative integer $d \leq n$ and define the rational function

$$
r_{n}[f](x)=\frac{\sum_{i=0}^{n-d} \lambda_{i}(x) p_{i}(x)}{\sum_{i=0}^{n-d} \lambda_{i}(x)},
$$

where $p_{i}(x)$ is the polynomial of degree $\leq d$ interpolating the $d+1$ values $f\left(x_{i}\right), \ldots$, $f\left(x_{i+d}\right)$, and

$$
\lambda_{i}(x)=\frac{(-1)^{i}}{\left(x-x_{i}\right) \cdots\left(x-x_{i+d}\right)} .
$$

This rational function interpolates and is analytic, has no real poles and, in the extreme case $d=n$, reduces to the polynomial interpolant of degree $\leq n$ of $f$. If $f \in C^{d+2}[a, b]$, the approximation rate is $O\left(h^{d+1}\right)$ as $h \rightarrow 0$ (under a bounded mesh ratio condition if $d=0$ ), where

$$
h=\max _{0 \leq i \leq n-1}\left(x_{i+1}-x_{i}\right)
$$

Received by the editor May 27, 2011 and, in revised form, February 6, 2012.

2010 Mathematics Subject Classification. Primary 65D05, 41A05, 41A20, 41A25; Secondary 65L12, 65D32.

Key words and phrases. Rational interpolation, barycentric form, Lebesgue function, condition.

The authors work was partly supported by the Swiss National Science Foundation under grant No. 200020-124779. 
is the mesh size. From Theorems 2 and 3 in [12] we see that $r_{n}$ reproduces polynomials of degree $\leq d$ and, if $n-d$ is odd, also reproduces polynomials of degree $d+1$.

For its evaluation, the rational interpolant (1.1) may be written in barycentric form [4, 7, 8,

$$
r_{n}[f](x)=\sum_{i=0}^{n} \frac{w_{i}}{x-x_{i}} f_{i} / \sum_{i=0}^{n} \frac{w_{i}}{x-x_{i}},
$$

where the formulas for the weights $w_{i}$ are derived in Section 4 of 12 and $f_{i}=f\left(x_{i}\right)$ are the given values of the function at the nodes.

The expression (1.2) constitutes a good interpolant and is also suited for the approximation of derivatives of functions [6], e.g., through finite differences [17, as well as for the approximation of integrals and antiderivatives (primitives) via quadrature rules [16. In particular, if the nodes may not be chosen at will and, e.g., need to be equispaced, (1.1) is a much better scheme for smooth approximation than its polynomial analogue. Numerical experiments show that the approximation quality with equispaced nodes is similar to that of splines. It was shown in 18 that it is not possible to construct a well-conditioned method with geometric convergence from data at equispaced nodes. The authors of that paper give a long list of various methods available at the present time for the interpolation in equispaced nodes; see also the references therein. The condition number of (1.1), which is its Lebesgue constant, is investigated in [9, 10]: it grows logarithmically with $n$ and exponentially with $d$.

In Section 2, we present an extension of the family of barycentric rational interpolants (1.2) in the equispaced case, before showing in Section 3 that this extension has a very small Lebesgue constant for any choice of $n$ and $d$. In Section 4 , we investigate some of its properties in various applications, such as the approximation of derivatives, integrals and antiderivatives. We conclude with numerical examples in Section 5 .

\section{Extension of the FloAter-Hormann FAMILY OF BARYCENTRIC RATIONAL INTERPOLANTS}

It is well known that polynomial interpolation at equispaced nodes is ill-conditioned. The Lebesgue constant $\Lambda_{n}$ grows exponentially with increasing degree $n$ [21, 26, 13] and amounts to about $3 \cdot 10^{12}$ for $n=50$ already. In floatingpoint arithmetic, the interpolation process will generally fail to converge whenever $\Lambda_{n}$ exceeds the reciprocal of the precision of the data, which is typically at most $10^{16}$. The rational interpolant (1.1) being a blend of polynomial interpolants of degree $\leq d$, increasing $d$ deteriorates the condition of the interpolation process [10]. For given data, $f_{0}, \ldots, f_{n}, r_{n}[f]$ may display high oscillations between the nodes towards the ends of the interval for a large $d$. The study of the Lebesgue function with equispaced nodes,

$$
\Lambda_{n}(x)=\sum_{i=0}^{n}\left|\frac{w_{i}}{x-x_{i}}\right| /\left|\sum_{i=0}^{n} \frac{w_{i}}{x-x_{i}}\right|,
$$

explains this behaviour independently of the interpolation data. It turns out that for given $d, \Lambda_{n}(x)$ has at most $d$ high oscillations at the ends and is much smaller in the remaining part of the interval; see Figure 1. This behaviour in the middle 

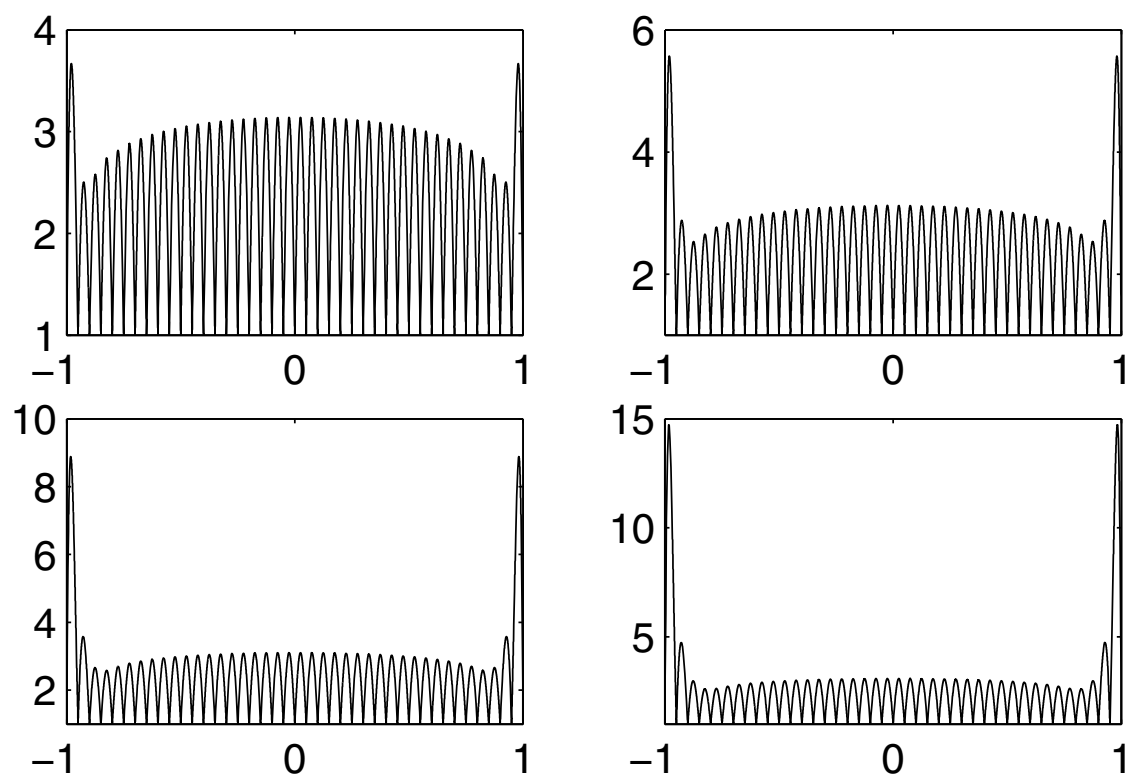

FiguRE 1. Lebesgue function for Floater-Hormann interpolation in equispaced nodes in $[-1,1]$ with $d=2,3,4,5$ and $n=40$.

part is very similar to that of the Lebesgue function for polynomial interpolation at Chebyshev points of the second kind, $x_{i}=\cos (i \pi / n)$. In order to improve the condition of (1.1), one may want to move the high oscillations out of the interval $[a, b]$. One possibility is to use the rational function only in the middle part of the interval, neglecting $d$ sub-intervals at each end of the interval. An alternative approach consists in adding $2 d$ new data values $\widetilde{f}_{-d}, \ldots, \widetilde{f}_{-1}$ and $\widetilde{f}_{n+1}, \ldots, \widetilde{f}_{n+d}$, corresponding to additional nodes $x_{-d}, \ldots, x_{-1}$ and $x_{n+1}, \ldots, x_{n+d}$, constructed from a smooth extension of $f$ beyond $x_{0}$ and $x_{n}$ using only the given data $f_{0}, \ldots, f_{n}$. The global data set is then interpolated by a rational function $r_{n+2 d}$ and evaluated only in the interval $[a, b]$. The procedure resembles that of adding "fictitious points" in finite difference approximation [13, Section 5.1] but it is not quite the same. Yet another approach, which also constructs data outside the interval and aims at regularizing interpolants of nonperiodic functions, is that of Fourier extensions; see [15] and the references therein.

Here we will look more closely at the second approach which adds $2 d$ new data values, since the first approach is trivial and implies a waste of data. The new data may be generated through a numerical Taylor expansion at each end of the interval, where the involved derivatives are approximated by one-sided finite difference formulas; see [13] for the polynomial version and [17] for the rational analogue based on the family of rational interpolants (1.1). To be precise, we choose positive integers $\widetilde{n} \ll n$ and $\widetilde{d} \leq \widetilde{n}$, and compute $r_{\widetilde{n}}^{(k)}[f]\left(x_{0}\right)$ and $r_{\widetilde{n}}^{(k)}[f]\left(x_{n}\right)$, the $k$ th derivatives at $x_{0}$ and $x_{n}$, respectively, of the rational interpolant of the values $f_{0}, \ldots, f_{\widetilde{n}}$ and $f_{n-\tilde{n}}, \ldots, f_{n}$, respectively, both with parameter $\widetilde{d}$, for $k=1, \ldots, \widetilde{d}$, provided $f$ is 
$2 \widetilde{d}+1$ times continuously differentiable [17. Then we set

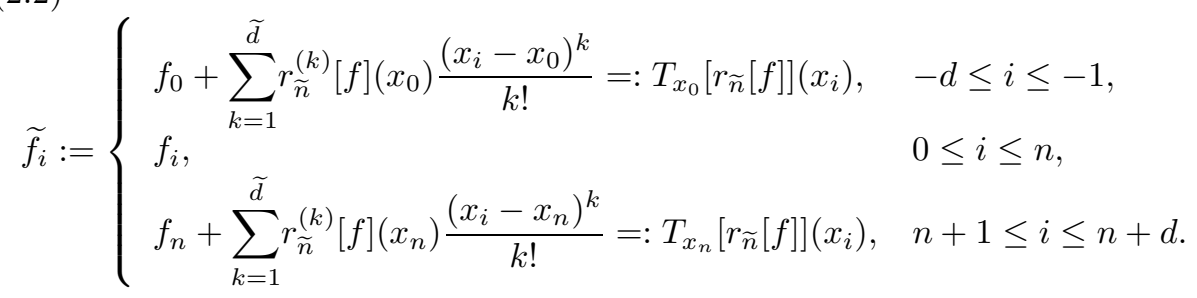

Our extension of the Floater-Hormann family of barycentric rational interpolants is then

$$
\widetilde{r}_{n}[f](x):=\sum_{i=-d}^{n+d} \frac{w_{i}}{x-x_{i}} \widetilde{f}_{i} / \sum_{i=-d}^{n+d} \frac{w_{i}}{x-x_{i}},
$$

where the barycentric weights $w_{i}$ are computed by means of the formula given in 12 for $n+2 d+1$ nodes. If $d=0$ the rational interpolant (1.2) remains unchanged; we may thus ignore this choice here.

The construction (2.2) only makes sense if the nodes are equispaced; we therefore suppose from now on that we are dealing with such nodes unless otherwise stated. Before giving the first result on $\widetilde{r}_{n}$, we introduce the following notation:

$$
D:=\min \{d, \widetilde{d}\} \text {. }
$$

Theorem 2.1. Suppose $n, d, \widetilde{n}<n$ and $\widetilde{d} \leq \widetilde{n}$ are positive integers and assume that $f \in C^{d+2}[a-d h, b+d h] \cap C^{2 \widetilde{d}+1}([a, a+\widetilde{n} h] \cup[b-\widetilde{n} h, b])$ is sampled at $n+1$ equispaced nodes in $[a, b]$. Then

$$
\left\|\widetilde{r}_{n}[f]-f\right\|:=\max _{x \in[a, b]}\left|\widetilde{r}_{n}[f](x)-f(x)\right| \leq C h^{D+1},
$$

where the constant $C$ only depends on $d, \widetilde{d}$ and derivatives of $f$. Moreover, $\widetilde{r}_{n}$ has no real poles and reproduces polynomials of degree $\leq \min \{\tilde{d}, d+1\}$ if $n+d$ is odd and $\leq D$ if $n+d$ is even.

Proof. Suppose we are given the exact data $f_{-d}, \ldots, f_{n+d}$. This allows us to form the rational interpolant $r_{n+2 d}[f]$ with parameter $d$, whose rate of convergence is $O\left(h^{d+1}\right)$. We let $x \in[a, b]$ and expand the absolute value of the error as

$$
\left|\widetilde{r}_{n}[f](x)-f(x)\right| \leq\left|\widetilde{r}_{n}[f](x)-r_{n+2 d}[f](x)\right|+\left|r_{n+2 d}[f](x)-f(x)\right| .
$$

The second term is bounded by $C h^{d+1}$, where $C$ is a constant depending only on $d$ and derivatives of $f$; see [12. (In what follows, we shall generically denote such constants by $C$.) The first term of the above right-hand side may be bounded from above by

$$
d ! h^{d+1} \sum_{\substack{-d \leq i \leq-1 \\ n+1 \leq i \leq n+d}} \frac{\left|w_{i}\right|}{\left|x-x_{i}\right|}\left|\tilde{f}_{i}-f_{i}\right|,
$$

where we treated the denominator of (2.3) as in [12]:

$$
\left|\sum_{i=-d}^{n+d} \frac{w_{i}}{x-x_{i}}\right|=\left|\sum_{i=-d}^{n} \lambda_{i}(x)\right| \geq \frac{1}{d ! h^{d+1}} .
$$


Let us look at $\left|\tilde{f}_{i}-f_{i}\right|$ for $i=-d, \ldots,-1$; for $i=n+1, \ldots, n+d$ the argument goes analogously. We denote by $T_{x_{0}}[f](x)$ the Taylor expansion of degree $\tilde{d}$ of $f$ about $x_{0}$. Then

$$
\begin{aligned}
\left|\tilde{f}_{i}-f_{i}\right| & \leq\left|\tilde{f}_{i}-T_{x_{0}}[f]\left(x_{i}\right)\right|+\left|T_{x_{0}}[f]\left(x_{i}\right)-f\left(x_{i}\right)\right| \\
& \leq \sum_{k=1}^{\widetilde{d}}\left|r_{\widetilde{n}}^{(k)}[f]\left(x_{0}\right)-f^{(k)}\left(x_{0}\right)\right| \frac{\left|x_{i}-x_{0}\right|^{k}}{k !}+\left|f^{(\widetilde{d}+1)}\left(\xi_{i}\right)\right| \frac{\left|x_{i}-x_{0}\right|^{\widetilde{d}+1}}{(\widetilde{d}+1) !},
\end{aligned}
$$

for some $\xi_{i} \in\left[x_{i}, x_{0}\right]$. It is shown in [17] that

$$
\left|r_{\widetilde{n}}^{(k)}[f]\left(x_{0}\right)-f^{(k)}\left(x_{0}\right)\right| \leq C h^{\widetilde{d}+1-k}, \quad 1 \leq k \leq \widetilde{d},
$$

and since $\left|x_{i}-x_{0}\right|^{k} \leq d^{k} h^{k}$, we have

$$
\left|\widetilde{f}_{i}-f_{i}\right| \leq C h^{\widetilde{d}+1}
$$

for $i=-d, \ldots,-1$ and $i=n+1, \ldots, n+d$. Finally, we notice from Section 4 in [12] that the weights $w_{i}$ in (2.6) are bounded as

$$
\left|w_{i}\right| \leq \frac{2^{d}}{d ! h^{d}}
$$

Since $x \in[a, b]$ and thus $\left|x-x_{i}\right| \geq h$, for $i=-d, \ldots,-1$ and $i=n+1, \ldots, n+d$, the claimed result (2.4) follows. The fact that $\widetilde{r}_{n}[f]$ in (2.3) has no real poles is trivial since the rational function (1.1) has no real poles for any number of nodes. Equation (2.5) with a polynomial $p$ instead of $f$ reveals that the first term vanishes if $\operatorname{deg}(p) \leq \widetilde{d}$ (see also (2.6) and (2.2) ), and that the second term equals 0 if $\operatorname{deg}(p) \leq d+1$ for $n+d$ odd and $\operatorname{deg}(p) \leq d$ for $n+d$ even, due to Theorem 2 in [12] for $n+2 d+1$ nodes.

The additional smoothness hypothesis in Theorem 2.1 and in the following results, as compared to those for (1.1), might be weakened if $f$ can be extended sufficiently smoothly from $[a, b]$ to $[a-d h, b+d h]$. On the other hand, additional smoothness properties of $f$ encourage the use of the presented interpolants, which are numerically better conditioned, as we will see in the next section.

\section{LEBESGUE CONSTANT}

In the case of a linear projection method, the Lebesgue constant may be defined as the norm of the approximation operator [19]. For the rational interpolant (1.2), it is given by

$$
\Lambda_{n}=\max _{x \in[a, b]} \Lambda_{n}(x),
$$

with $\Lambda_{n}(x)$ from (2.1), and analogously for (2.3), which may be denoted by $\widetilde{\Lambda}_{n}$. The Lebesgue constant $\Lambda_{n}$ is the condition number of the associated interpolation method. Let us suppose that every $f_{i}$ is given with an error at most $\varepsilon$ in absolute value, $\varepsilon>0$. Then the norm of the difference between the interpolant $r_{n}[f, \varepsilon]$ of the perturbed data and $r_{n}[f]$ is bounded as

$$
\left\|r_{n}[f, \varepsilon]-r_{n}[f]\right\| \leq \max _{x \in[a, b]} \sum_{i=0}^{n}\left|\frac{w_{i}}{x-x_{i}}\right| \varepsilon /\left|\sum_{i=0}^{n} \frac{w_{i}}{x-x_{i}}\right|=\varepsilon \Lambda_{n},
$$

where equality holds if all the perturbations equal $\varepsilon$ in absolute value and if their signs are such that all the terms in the sum in the numerator of $r_{n}[f, \varepsilon]-r_{n}[f]$ 
are positive. Moreover, $\Lambda_{n}$ gives information about the quantity by which the interpolant may grow between the nodes when interpolating the values \pm 1 . For the family of Floater-Hormann interpolants at equispaced nodes, it is shown in [10] that, for $d \geq 1, \Lambda_{n}$ roughly goes as follows:

$$
\frac{2^{d-2}}{d+1} \ln \left(\frac{n}{d}-1\right) \leq \Lambda_{n} \leq 2^{d-1}(2+\ln n)
$$

For $d=0$, the same bounds as for $d=1$ are valid; see also 9, 14. We see that $\Lambda_{n}$ grows logarithmically with $n$, but exponentially with $d$. This is one reason why one should preferably use rather small values of $d$ when interpolating at equispaced nodes, and not increase $d$ with $n$, at least not too fast.

If the exact values of $f$ are given as $\widetilde{f}_{i}$ at $x_{i}$ for $i=-d, \ldots,-1$ and $i=$ $n+1, \ldots, n+d$, then the Lebesgue function associated with extended FloaterHormann interpolation is the analogue of (2.1). Since these $\tilde{f}_{i}$ may be obtained differently than presented here, and as we do not use the interpolants outside the interval $[a, b]$, we may ignore uncertainties in these values obtained from (2.2) and consider the analogue of (2.1) in $[a, b]$ for the study of the condition. With this interpretation, the extended rational interpolants (2.3) have Lebesgue constants that grow logarithmically in $n$ and $d$, as the following theorem shows.

Theorem 3.1. For positive integers $n$ and $d$, the Lebesgue constant $\widetilde{\Lambda}_{n}$ for the basis of the extended barycentric rational interpolants at $n+1$ equispaced nodes in the interval $[a, b]$ is bounded from above as

$$
\widetilde{\Lambda}_{n} \leq \frac{2^{d-1}}{d ! \sum_{i=0}^{d-1} \frac{1}{\prod_{j=i}^{d-2}\left(d-\frac{1}{2}-j\right) \prod_{\ell=1}^{i}\left(\ell+\frac{1}{2}\right)}}(2+\ln (n+2 d)) .
$$

REMARK: The leading quotient in (3.1) is $\leq 1$ for all positive $d$ and for $d \geq 5$ it almost becomes constant, so that

$$
\widetilde{\Lambda}_{n} \leq 0.65(2+\ln (n+2 d)), \quad \text { for } d \geq 5 .
$$

This means that $\widetilde{\Lambda}_{n}$ grows merely logarithmically with $n$ and $d$, and that its upper bound is very close to the $1+\frac{2}{\pi} \log (n+1)$ bound for polynomial interpolation at Chebyshev points of the first kind, $x_{i}=\cos (\pi / 2 \cdot(2 i+1) /(n+1))$, and those of the second kind [1].

Proof. This proof uses some tools from [10]. If $x=x_{k}$ for $k=0, \ldots, n$, then $\widetilde{\Lambda}_{n}(x)=1$, in view of the interpolation property. Suppose that $x_{k}<x<x_{k+1}$ for $k \in\{0, \ldots, n-1\}$. We multiply the numerator and the denominator of the Lebesgue function associated with (2.3) by $\left(x-x_{k}\right)\left(x_{k+1}-x\right)$ and take (2.9) into account to obtain

$$
\widetilde{\Lambda}_{n}(x) \leq \frac{2^{d}}{d ! h^{d}} \frac{\sum_{i=-d}^{n+d}\left|\frac{1}{x-x_{i}}\right|\left(x-x_{k}\right)\left(x_{k+1}-x\right)}{\left|\sum_{i=-d}^{n+d} \frac{w_{i}}{x-x_{i}}\right|\left(x-x_{k}\right)\left(x_{k+1}-x\right)}=\frac{2^{d}}{d ! h^{d}} \frac{N(x)}{D(x)} .
$$

Let us first look at the numerator:

$$
\begin{aligned}
N(x) & =x_{k+1}-x_{k}+\left(x-x_{k}\right)\left(x_{k+1}-x\right)\left(\sum_{i=-d}^{k-1} \frac{1}{x-x_{i}}+\sum_{i=k+2}^{n+d} \frac{1}{x_{i}-x}\right) \\
& \leq h+\left(\frac{h}{2}\right)^{2}\left(\sum_{i=-d}^{k-1} \frac{1}{x_{k}-x_{i}}+\sum_{i=k+2}^{n+d} \frac{1}{x_{i}-x_{k+1}}\right) .
\end{aligned}
$$


Since the nodes $x_{i}$ are equispaced, the first sum simplifies to $\sum_{i=1}^{k+d} \frac{1}{i h}$, which is less than $\ln (2 k+2 d+1) / h$, and analogously for the second sum. An upper bound for the numerator now follows:

$$
\begin{aligned}
N(x) & \leq h+\frac{h}{4} \ln ((2 k+2 d+1)(2 n+4 d-(2 k+2 d+1))) \\
& \leq h+\frac{h}{4} \ln \left(\frac{2 n+4 d}{2}\right)^{2}=h+\frac{h}{2} \ln (n+2 d) .
\end{aligned}
$$

For our study of the denominator $D(x)$ we recall some results from [12] and adapt them to our setting. To begin with, we rewrite $D(x)$ using the original definition of the denominator of the rational interpolant,

$$
D(x)=\left(x-x_{k}\right)\left(x_{k+1}-x\right)\left|\sum_{i=-d}^{n} \lambda_{i}(x)\right| .
$$

With the notations

$$
\mu_{i}(x):=(-1)^{n+d} \lambda_{i}(x) \prod_{j=-d}^{n+d}\left(x-x_{j}\right)
$$

and

$$
s(x):=\sum_{i=-d}^{n+d} \mu_{i}(x)
$$

it was shown in 12 that

$$
s(x)>0 \quad \text { and } \quad s(x) \geq \sum_{i=k-d+1}^{k} \mu_{i}(x)=: s_{2}(x)>0 .
$$

From these results, we may proceed with

$$
D(x) \geq\left(x-x_{k}\right)\left(x_{k+1}-x\right) \frac{s_{2}(x)}{\prod_{j=-d}^{n+d}\left|x-x_{j}\right|},
$$

whose right-hand side yields after cancellations

$$
\sum_{i=k-d+1}^{k} \tilde{\lambda}_{i}(x) \quad \text { with } \quad \tilde{\lambda}_{i}(x):=\frac{1}{\prod_{j=i}^{k-1}\left(x-x_{j}\right) \prod_{\ell=k+2}^{i+d}\left(x_{\ell}-x\right)} .
$$

We may now deduce that the last sum has exactly one minimum in $\left(x_{k}, x_{k+1}\right)$ at $x=x_{k}+\frac{h}{2}$. It is not difficult to see that the sum, as a function of $x$, is symmetric about $x_{k}+\frac{h}{2}$. Moreover, its derivative

$$
\frac{d}{d x} \sum_{i=k-d+1}^{k} \tilde{\lambda}_{i}(x)=\sum_{i=k-d+1}^{k} \tilde{\lambda}_{i}(x)\left(-\sum_{j=i}^{k-1} \frac{1}{x-x_{j}}+\sum_{\ell=k+2}^{i+d} \frac{1}{x_{\ell}-x}\right)
$$

is negative in $\left(x_{k}, x_{k}+\frac{h}{2}\right)$, since the $m$ th term $\left(m=1, \ldots,\left\lfloor\frac{d}{2}\right\rfloor\right)$ in the sum in the right-hand side is negative and strictly larger in absolute value than the $(d-$ $m$ )th term, which is positive. The middle term, if it exists, is also negative in the considered interval. A similar argument shows that (3.4) is strictly positive in 

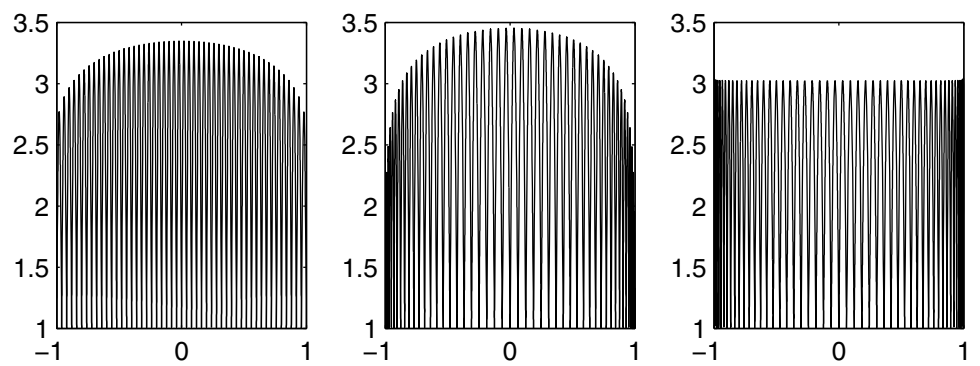

FiguRE 2. Lebesgue function with $n=50$ for extended FloaterHormann interpolation in equispaced nodes with $d=3$ (left), for polynomial interpolation in Chebyshev points of the second kind (center) and first kind (right).

$\left(x_{k}+\frac{h}{2}, x_{k+1}\right)$ and it is easy to see that it vanishes at $x=x_{k}+\frac{h}{2}$. We have thus established that

$$
D(x) \geq h^{-d+1} \sum_{i=k-d+1}^{k} \frac{1}{\prod_{j=i}^{k-1}\left(k+\frac{1}{2}-j\right) \prod_{\ell=k+2}^{i+d}\left(\ell-k-\frac{1}{2}\right)} .
$$

After a rearrangement of the indices, this gives a bound on the denominator:

$$
D(x) \geq h^{-d+1} \sum_{i=0}^{d-1} \frac{1}{\prod_{j=i}^{d-2}\left(d-\frac{1}{2}-j\right) \prod_{\ell=1}^{i}\left(\ell+\frac{1}{2}\right)} .
$$

Together with (3.2), the bounds on the numerator (3.3) and denominator (3.5) yield the upper bound (3.1) for the Lebesgue constant.

To conclude this section, we take a look at the behaviour of the Lebesgue functions and constants associated with the various methods. In Figure 2 we compare graphically the Lebesgue functions associated with extended Floater-Hormann interpolation at equispaced nodes with $d=3$ and polynomial interpolation at Chebyshev points of the second and first kinds, all three with $n=50$. The maximal height of the respective functions, i.e., the Lebesgue constants, are very close to each other; the shapes of the first two are similar. This picture does not fundamentally change with a different choice of $n$ or $d$. From Figure 3 we see that, already with $d=8$, the difference in magnitude between the Lebesgue constants corresponding to the original Floater-Hormann interpolation and its extended counterpart is striking. This is further stressed with Figure 4 which shows that the Lebesgue constant associated with Floater-Hormann interpolation grows exponentially fast with $d$ whereas that associated with the extended family increases very slowly from 4.19 with $d=1$ to 4.26 with $d=25$.

\section{Applications}

As already stated in the Introduction, the family of rational interpolants (1.1) may be used in applications such as the approximation of derivatives of a function, its integral or antiderivatives [6, 16, 17]. In this section, we will investigate the behaviour of (2.3) regarding these applications for functions sampled at equispaced nodes. The rates of convergence stay roughly the same as with the original family 


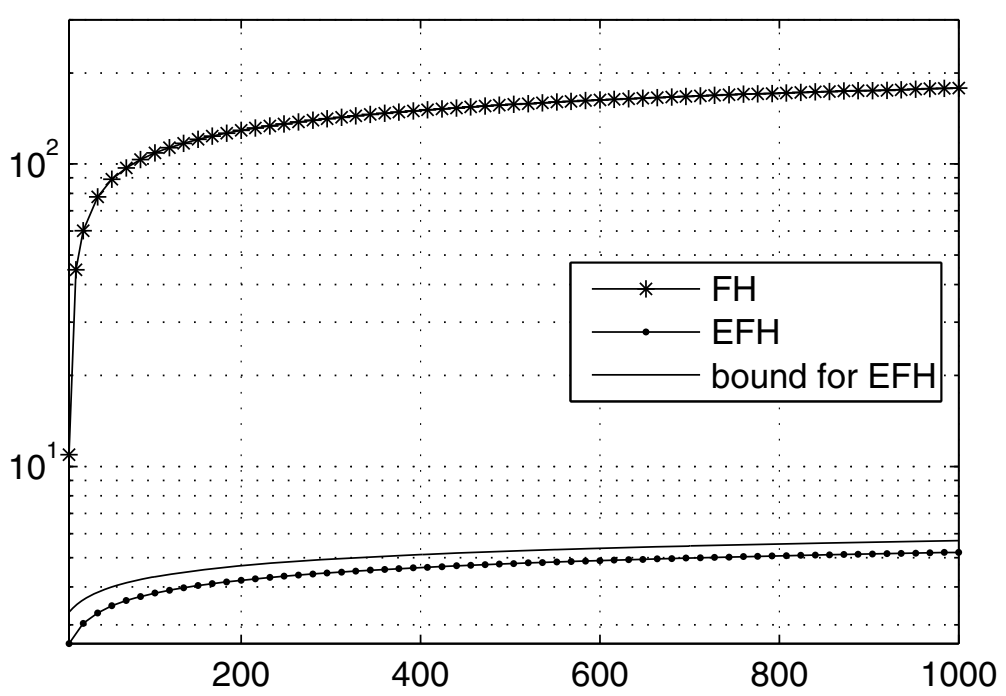

Figure 3. Lebesgue constants associated with Floater-Hormann $(\mathrm{FH})$ and extended Floater-Hormann (EFH) interpolation with $d=8$ and $8 \leq n \leq 1000$, together with the upper bound on the EFH Lebesgue constant.

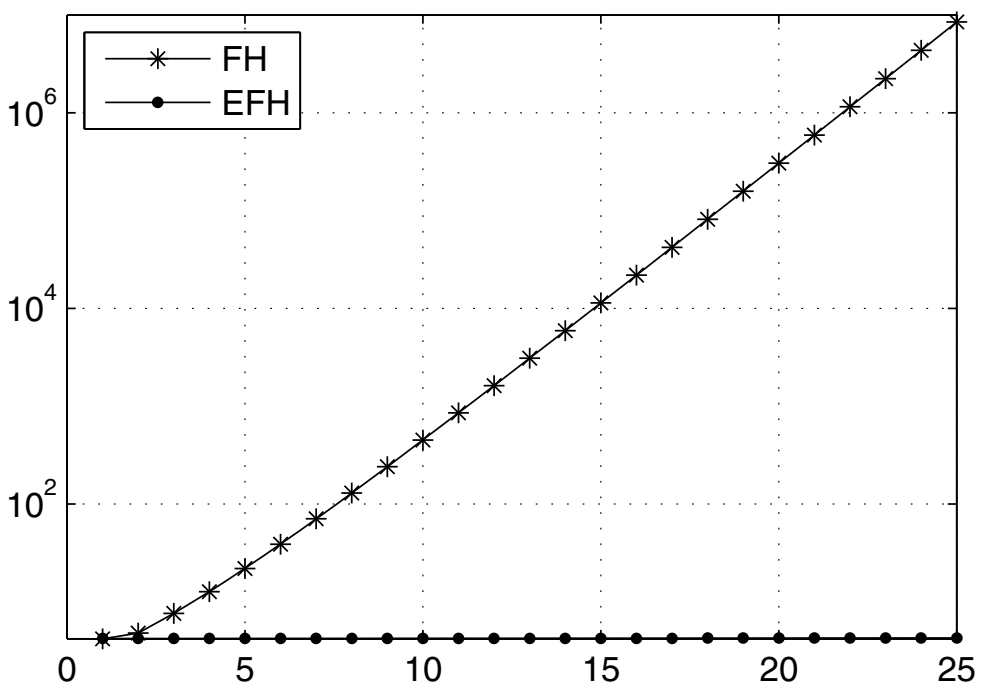

FiguRE 4. Lebesgue constants associated with Floater-Hormann $(\mathrm{FH})$ and extended Floater-Hormann $(\mathrm{EFH})$ interpolation in equispaced nodes with $n=200$ and $1 \leq d \leq 25$. 
of interpolants, but the constants involved in the error bounds are smaller in many cases, as documented in Section 5 .

4.1. Differentiation. Let us begin with the approximation of derivatives at the nodes $a=x_{0}, x_{1}, \ldots, x_{n}=b$.

Theorem 4.1. Suppose $n, d, \widetilde{n}<n, \widetilde{d} \leq \widetilde{n}$ and $k \leq D$ are positive integers and assume that $f \in C^{d+1+k}[a-d h, b+d h] \cap C^{2 \widetilde{d}+1}([a, a+\widetilde{n} h] \cup[b-\widetilde{n} h, b])$ is sampled at $n+1$ equispaced nodes $a=x_{0}<x_{1}<\ldots<x_{n}=b$. Then

$$
\left|\widetilde{r}_{n}^{(k)}[f]\left(x_{j}\right)-f^{(k)}\left(x_{j}\right)\right| \leq C h^{D+1-k}, \quad-d \leq j \leq n+d,
$$

where the constant $C$ only depends on $d, \widetilde{d}, k$ and derivatives of $f$.

Proof. Suppose again we are given the exact data $f_{-d}, \ldots, f_{n+d}$. We split the absolute value of the $k$ th derivative of the interpolation error at $x=x_{j},-d \leq j \leq$ $n+d$, into two parts,

$$
\begin{aligned}
\left|\widetilde{r}_{n}^{(k)}[f]\left(x_{j}\right)-f^{(k)}\left(x_{j}\right)\right| \leq & \left|\widetilde{r}_{n}^{(k)}[f]\left(x_{j}\right)-r_{n+2 d}^{(k)}[f]\left(x_{j}\right)\right| \\
& +\left|r_{n+2 d}^{(k)}[f]\left(x_{j}\right)-f^{(k)}\left(x_{j}\right)\right| .
\end{aligned}
$$

The second term is bounded by $C h^{d+1-k}$ for $j=-d, \ldots, n+d$ as an immediate consequence of Theorem 1 in [17, which states that such a bound holds for the absolute value of the $k$ th derivative of the interpolation error of (1.1) at the nodes. It therefore remains to bound the first term. For $j=0, \ldots, n$ we may consider the expression inside the absolute values and write it as

$$
\left.\frac{d^{k-1}}{d x^{k-1}}\right|_{x=x_{j}} \frac{\sum_{i=-d}^{-1} \frac{w_{i}}{x-x_{i}}\left(\widetilde{f}_{i}-f_{i}\right)+\sum_{i=n+1}^{n+d} \frac{w_{i}}{x-x_{i}}\left(\tilde{f}_{i}-f_{i}\right)}{g_{j}(x)} k,
$$

where

$$
g_{j}(x):=\left(x-x_{j}\right) \sum_{i=-d}^{n} \lambda_{i}(x) .
$$

Expression (4.2) is obtained by comparing coefficients in the Taylor expansions at $x=x_{j}$ of the interpolation error and this error divided by $\left(x-x_{j}\right)$; see [17. Let us call $A(x)$ the numerator of the quotient in (4.2). Applying the Leibniz rule and dividing by $k$ then yields

$$
\left.\sum_{\ell=0}^{k-1}\left(\begin{array}{c}
k-1 \\
\ell
\end{array}\right) A^{(k-1-\ell)}(x)\left(g_{j}(x)\right)^{-1}\right)\left.^{(\ell)}\right|_{x=x_{j}} .
$$

The $\ell$ th derivative of the reciprocal of $g_{j}(x)$ is bounded by $C h^{d-\ell}$ at $x=x_{j}$, as shown in the proof of Theorem 1 in [17. It remains to deal with the absolute values of the derivatives of $A(x)$ at $x=x_{j}$. We observe that for $0 \leq \ell \leq k-1$,

$$
\left|A^{(k-1-\ell)}\left(x_{j}\right)\right| \leq(k-1-\ell) ! \sum_{\substack{d \leq i \leq-1 \\ n+1 \leq i \leq n+d}}\left|w_{i}\right|\left|x_{j}-x_{i}\right|^{\ell-k}\left|\tilde{f}_{i}-f_{i}\right|,
$$

which we bound from above by $C h^{\widetilde{d}+1-d-k+\ell}$, using (2.8) and (2.9). This, together with the bound on the $\ell$ th derivative of the reciprocal of $g_{j}(x)$ in the absolute value of (4.2), gives the bound $C h^{\widetilde{d}+1-k}$ for the first term in (4.1) in the present case. 
For $j=-d, \ldots,-1$ and $j=n+1, \ldots, n+d$ we write the expression inside the absolute values in the first term in (4.1) as

$$
\left.\frac{d^{k}}{d x^{k}}\right|_{x=x_{j}} \frac{\sum_{i=-d}^{-1} w_{i} \frac{x-x_{j}}{x-x_{i}}\left(\tilde{f}_{i}-f_{i}\right)+\sum_{i=n+1}^{n+d} w_{i} \frac{x-x_{j}}{x-x_{i}}\left(\tilde{f}_{i}-f_{i}\right)}{g_{j}(x)} .
$$

With the Leibniz rule and the observation that the $\ell$ th derivative, $1 \leq \ell \leq k$, at $x=x_{j}$ of the numerator $\widetilde{A}(x)$ of the quotient in (4.3) is bounded as

$$
\left|\widetilde{A}^{(\ell)}\left(x_{j}\right)\right| \leq \ell ! \sum_{\substack{d \leq i \leq-1 \\ n+1 \leq i \leq n+d \\ i \neq j}}\left|w_{i}\right|\left|x_{j}-x_{i}\right|^{-\ell}\left|\tilde{f}_{i}-f_{i}\right|
$$

and that $\left|\widetilde{A}\left(x_{j}\right)\right| \leq C h^{\widetilde{d}+1-d}$, it follows that the first term in (4.1) is also bounded by $C h^{\widetilde{d}+1-k}$ for $j=-d, \ldots,-1$ and $j=n+1, \ldots, n+d$.

This result leads us to define extended rational finite difference methods (ERFD), which are FD methods [13, 22] derived from the extended family of rational interpolants (2.3) for the approximation at the nodes $x_{i}, 0 \leq i \leq n$, of the $k$ th derivative of a sufficiently smooth function,

$$
\left.\frac{d^{k} f}{d x^{k}}\right|_{x=x_{i}} \approx \frac{d^{k}}{d x^{k}} \widetilde{r}_{n}[f]\left(x_{i}\right)=\sum_{j=-d}^{n+d} D_{i j}^{(k)} \widetilde{f}_{j}=: \widetilde{f}_{i}^{(k)} .
$$

The weights $D_{i j}^{(k)}$ are the elements from the $(d+1)$ st to the $(n+d+1)$ st row of the $(n+2 d+1) \times(n+2 d+1)$ differentiation matrix $D^{(k)}$ (the indices are shifted according to the indices of the extended set of nodes). These matrices are constructed [2, 3, 23, 24] from Proposition 11 in [20; see also [17]. The weights for the first order left one-sided ERFD approximation, i.e., that at $x=x_{0}$, satisfy

$$
\frac{1}{2^{d}(b-a+d h)} \leq\left|D_{0 j}^{(1)}\right| \leq \frac{1}{h}, \quad j=-d, \ldots, n+d,
$$

for all admissible $n$ and $d$.

For the approximation of the $k$ th derivative of a function $f$ at intermediate points $x \in[a, b]$, we suggest, similarly as in [17, to interpolate the approximations at the nodes $\widetilde{f}_{i}^{(k)}$ from (4.4) by a rational function (2.3):

$$
\widetilde{R}_{n}^{(k)}[f](x):=\sum_{i=-d}^{n+d} \frac{w_{i}}{x-x_{i}} \widetilde{f}_{i}^{(k)} / \sum_{i=-d}^{n+d} \frac{w_{i}}{x-x_{i}} .
$$

This formula is less expensive to evaluate outside the nodes than the exact derivative of $\widetilde{r}_{n}[f]$ and, as we shall now see, it follows from Theorems 3.1 and 4.1 that its rate of convergence towards the exact derivative of $f$ throughout the interval $[a, b]$ is almost the same as the $O\left(h^{D+1-k}\right)$ rate at the nodes from the latter theorem.

Proposition 4.2. Suppose $n, d, \widetilde{n}<n, \widetilde{d} \leq \widetilde{n}$ and $k \leq D$ are positive integers and assume that $f \in C^{d+2+k}[a-d h, b+d h] \cap C^{2 \widetilde{d}+1}([a, a+\widetilde{n} h] \cup[b-\widetilde{n} h, b])$ is sampled at $n+1$ equispaced nodes $a=x_{0}<x_{1}<\ldots<x_{n}=b$. Then

$$
\left\|\widetilde{R}_{n}^{(k)}[f]-f^{(k)}\right\|=\max _{x \in[a, b]}\left|\widetilde{R}_{n}^{(k)}[f](x)-f^{(k)}(x)\right| \leq C h^{D+1-k}(1+\ln (n+2 d)),
$$

where the constant $C$ only depends on $d, \widetilde{d}, k$ and derivatives of $f$. 
Proof. The function $f$ is supposed to belong to $C^{d+2+k}[a-d h, b+d h]$. Its $k$ th derivative may be interpolated at the nodes $x_{-d}, \ldots, x_{n+d}$ with approximation rate $O\left(h^{d+1}\right)$ by the rational function $r_{n+2 d}\left[f^{(k)}\right]$ with parameter $d$ from (1.1). For $x \in[a, b]$, we expand the absolute value of the error as

$$
\begin{aligned}
\left|\widetilde{R}_{n}^{(k)}[f](x)-f^{(k)}(x)\right| \leq & \frac{\sum_{i=-d}^{n+d} \frac{\left|w_{i}\right|}{\left|x-x_{i}\right|}\left|\widetilde{f}_{i}^{(k)}-f^{(k)}\left(x_{i}\right)\right|}{\left|\sum_{i=-d}^{n+d} \frac{w_{i}}{x-x_{i}}\right|} \\
& +\left|r_{n+2 d}\left[f^{(k)}\right](x)-f^{(k)}(x)\right| .
\end{aligned}
$$

From Theorem 3.1 we see that the first term is bounded by

$$
\widetilde{\Lambda}_{n} \max _{-d \leq i \leq n+d}\left|\widetilde{f}_{i}^{(k)}-f^{(k)}\left(x_{i}\right)\right|,
$$

which is less than $C h^{D+1-k}(1+\ln (n+2 d))$; see also Theorem 4.1. This, combined with the $O\left(h^{d+1}\right)$ bound on the second term in (4.6), gives the result.

4.2. Quadrature and approximation of antiderivatives. Suppose we want to approximate the integral of an integrable function $f$ over the interval $[a, b]$, where it is sampled at $n+1$ equispaced nodes. For data available at equispaced nodes and at a few additional points, quadrature rules obtained from applying endpoint corrections to the trapezoidal rule are derived in [1] and in the references therein. In [16] a method called direct rational quadrature (DRQ), based on the original family (1.1), is presented. Let us follow the same approach for the extended family (2.3), i.e.,

$$
\int_{a}^{b} f(x) d x \approx \int_{a}^{b} \widetilde{r}_{n}[f](x) d x=\int_{a}^{b} \frac{\sum_{i=-d}^{n+d} \frac{w_{i}}{x-x_{i}} \tilde{f}_{i}}{\sum_{\ell=-d}^{n+d} \frac{w_{\ell}}{x-x_{\ell}}} d x=\sum_{i=-d}^{n+d} \omega_{i} \widetilde{f}_{i},
$$

where

$$
\omega_{i}:=\int_{a}^{b} \frac{\frac{w_{i}}{x-x_{i}}}{\sum_{\ell=-d}^{n+d} \frac{w_{\ell}}{x-x_{\ell}}} d x .
$$

The integrand in the definition of the quadrature weights $\omega_{i}$ may be evaluated at every point in the interval $[a, b]$. For this reason we approximate the integral giving $\omega_{i}$ by an efficient quadrature rule, e.g., Gauss-Legendre, and call the approximated weights $\omega_{i}^{\mathcal{D}}$. The corresponding method, the extended direct rational quadrature (EDRQ), then reads

$$
\int_{a}^{b} f(x) d x \approx \sum_{i=-d}^{n+d} \omega_{i}^{\mathcal{D}} \widetilde{f}_{i}
$$

An explicit knowledge of the weights $\omega_{i}^{\mathcal{D}}$ is not always necessary in practice: as $\widetilde{r}_{n}[f]$ is analytic, it is sufficient to apply a quadrature rule on the interpolant to directly compute (4.8). The following theorem gives the main properties of EDRQ.

Theorem 4.3. Suppose $n, d, \widetilde{n}<n$ and $\widetilde{d} \leq \widetilde{n}$ are positive integers and assume that $f \in C^{d+3}[a-d h, b+d h] \cap C^{2 \widetilde{d}+1}([a, a+\widetilde{n} h] \cup[b-\widetilde{n} h, b])$ is sampled at $n+1$ equispaced nodes in $[a, b]$. Let the quadrature weights $\omega_{i}$ in (4.7) be approximated 
by a linear quadrature rule $Q$ converging at least at the rate $O\left(h^{d+2}\right)$. Then

$$
\left|\sum_{i=-d}^{n+d} \omega_{i}^{\mathcal{D}} \tilde{f}_{i}-\int_{a}^{b} f(x) d x\right| \leq C h^{D+2} \ln n
$$

where the constant $C$ only depends on $d, \widetilde{d}$ and derivatives of $f$. Moreover, if the quadrature rule $Q$ is symmetric and has polynomial degree of precision at least $\min \{d+1, \widetilde{d}\}$, then the resulting EDRQ rule is symmetric and its polynomial degree of precision is $\min \{d+1, \widetilde{d}\}$ if $n+d$ is odd and $D$ if $n+d$ is even.

Proof. We begin with splitting the absolute value of the quadrature error into two parts,

$$
\begin{aligned}
\left|\sum_{i=-d}^{n+d} \omega_{i}^{\mathcal{D}} \tilde{f}_{i}-\int_{a}^{b} f(x) d x\right| \leq & \left|\sum_{i=-d}^{n+d} \omega_{i}^{\mathcal{D}} \widetilde{f}_{i}-\int_{a}^{b} \widetilde{r}_{n}[f](x) d x\right| \\
& +\left|\int_{a}^{b}\left(\widetilde{r}_{n}[f](x)-f(x)\right) d x\right|
\end{aligned}
$$

The first part is bounded by $C h^{D+2}$ because of the rate of convergence of $Q$. We subdivide the second part into

$\int_{a}^{x_{1}}\left|\widetilde{r}_{n}[f](x)-f(x)\right| d x+\left|\int_{x_{1}}^{x_{n-1}}\left(\widetilde{r}_{n}[f](x)-f(x)\right) d x\right|+\int_{x_{n-1}}^{b}\left|\widetilde{r}_{n}[f](x)-f(x)\right| d x$.

The sum of the first and last terms is bounded by $2 h\left\|\widetilde{r}_{n}[f]-f\right\|$, which is less than or equal to $C h^{D+2}$ by Theorem 2.1. To treat the middle term, we assume that the values of the function $f$ are given at all the nodes $x_{-d}, \ldots, x_{n+d}$ and we interpolate it by the rational function (1.1) with parameter $d$ at these nodes. After adding and subtracting $r_{n+2 d}[f](x)$ in the argument, simplifying and writing the interpolation error as in [12, it becomes

$$
\begin{aligned}
\sum_{\substack{-d \leq i \leq-1 \\
n+1 \leq i \leq n+d}} w_{i}\left(\tilde{f}_{i}-f_{i}\right) \int_{x_{1}}^{x_{n-1}} \frac{1}{\left(x-x_{i}\right) \sum_{\ell=-d}^{n} \lambda_{\ell}(x)} d x \\
+\int_{x_{1}}^{x_{n-1}} \frac{\sum_{i=-d}^{n}(-1)^{i} f\left[x_{i}, \ldots, x_{i+d}, x\right]}{\sum_{\ell=-d}^{n} \lambda_{\ell}(x)} d x .
\end{aligned}
$$

It is shown in the proof of Theorem 2 in [16 that the absolute value of the second term is bounded by $C h^{d+2}$ as it corresponds to the integral of the interpolation error over the middle part of the interval of interpolation, i.e., the part without the first and last $d+1$ sub-intervals. The factors $\left(x-x_{i}\right)$ in the integrand of the first part of the above expression do not change sign in the interval $\left[x_{1}, x_{n-1}\right]$ since $i \notin\{1, \ldots, n-1\}$; the application of the mean value theorem for integrals yields

$$
\sum_{\substack{-d \leq i \leq-1 \\ n+1 \leq i \leq n+d}} w_{i}\left(\widetilde{f}_{i}-f_{i}\right) \frac{1}{\sum_{\ell=-d}^{n} \lambda_{\ell}\left(\xi_{i}\right)} \int_{x_{1}}^{x_{n-1}} \frac{1}{x-x_{i}} d x
$$

for some $\xi_{i} \in\left[x_{1}, x_{n-1}\right]$. The claimed error bound now follows with (2.7), (2.8) and (2.9).

The symmetry of EDRQ follows directly from Theorem 4 in [16], which shows that the integrand in the $m$ th quadrature weight is symmetric to that in the $(n+1-$ 
$m)$ th weight with respect to the midpoint of the interval. The polynomial degree of precision follows from the fact that the extended rational interpolants reproduce polynomials of the claimed degree; see Theorem 2.1 .

Indirect rational quadrature (IRQ), a method based on linear barycentric rational interpolation for the approximation of an antiderivative of such a function, is presented in [16] as well. With the extended family of rational interpolants (2.3), the method (EIRQ) becomes the following: we approximate $\int_{a}^{x} f(y) d y$ by the rational function

$$
\widetilde{r}_{n}[u](x),
$$

interpolating the result $u$ of a collocation [5] at the nodes $x_{-d}, \ldots, x_{n+d}$ applied to the initial value problem

$$
\frac{d}{d x} \widetilde{r}_{n}[u](x) \approx \widetilde{f}(x), \quad u_{0}=0, \quad x \in[a-d h, b+d h],
$$

where $\tilde{f}(x)$ is a function with values $\tilde{f}_{-d}, \ldots, \widetilde{f}_{n+d}$ at the nodes as defined in Section 2 In other words, we solve the system of $n+2 d$ equations

$$
\sum_{\substack{j=-d \\ j \neq 0}}^{n+d} \widetilde{D}_{i j}^{(1)} u_{j}=\widetilde{f}_{i}, \quad i=-d, \ldots,-1,1, \ldots, n+d,
$$

with $\widetilde{D}^{(1)}$ the first order differentiation matrix defined in Section 4.1, without its $(d+1)$ st row and column, and insert the so-obtained $u_{-d}, \ldots, u_{n+d}$ into (4.9). Note that $u_{n}$ gives an approximation of the integral of $f$ over $[a, b]$ and that the approximation (4.9) is analytic.

\section{Numerical RESUlts}

Let us now look at a few numerical examples illustrating the results and remarks from Sections 2 to 4 . The examples document the error behaviour of extended Floater-Hormann interpolation from equispaced samples and of the applications in this work, i.e., the approximation of derivatives, integrals and antiderivatives. We compare it to original Floater-Hormann interpolation with the same value of the parameter $d$ and to B-splines of order $d+1$ obtained with the spapi command from the MatLAB curve-fitting toolbox. In all the tests, the values of $n$ are even and the parameters for the extended Floater-Hormann interpolants remain fixed at $\widetilde{d}=7$ and $\widetilde{n}=11$. The experimental convergence rates may be read from the slopes in the logarithmic plots. The errors are computed as the maximum absolute values of the differences between the interpolant and the exact function at 2000 equispaced points in the interval $[a, b]$.

Figure 5 shows the interpolation of Runge's function $f_{1}(x):=1 /\left(1+x^{2}\right)$ in the interval $[-5,5]$ for the three interpolants with theoretical convergence rate $O\left(h^{5}\right)$. The slopes in the error curves are almost identical for large enough $n$, but the errors in the rational interpolants are much smaller than those in the spline. With the interpolation of $\sin (x)$ the picture is similar, only the values of the errors are closer together; we omit the corresponding plot.

The next example deals with the conditioning of the interpolation process. It is well known that, due to the Runge phenomenon, the polynomial interpolant of $f_{1}$, sampled at equispaced nodes, diverges as $n$ increases. Suppose the perturbation 


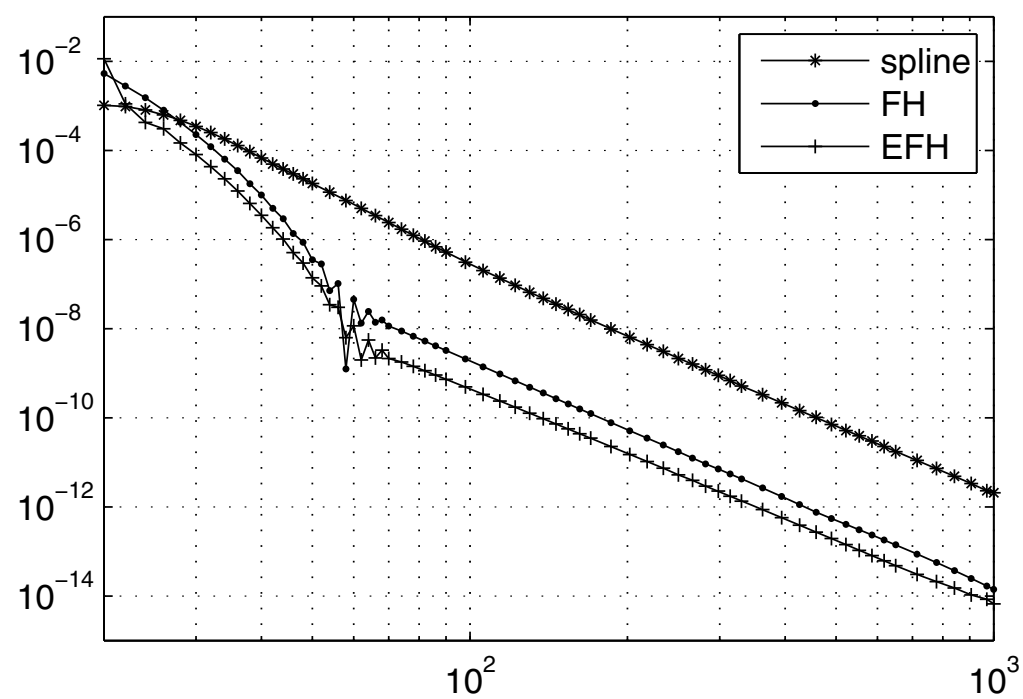

FiguRE 5. Error behaviour of spline, Floater-Hormann (FH) and extended Floater-Hormann $(\mathrm{EFH})$ interpolation of $f_{1}$ in $[-5,5]$ with $d=4$ and $20 \leq n \leq 1000$.

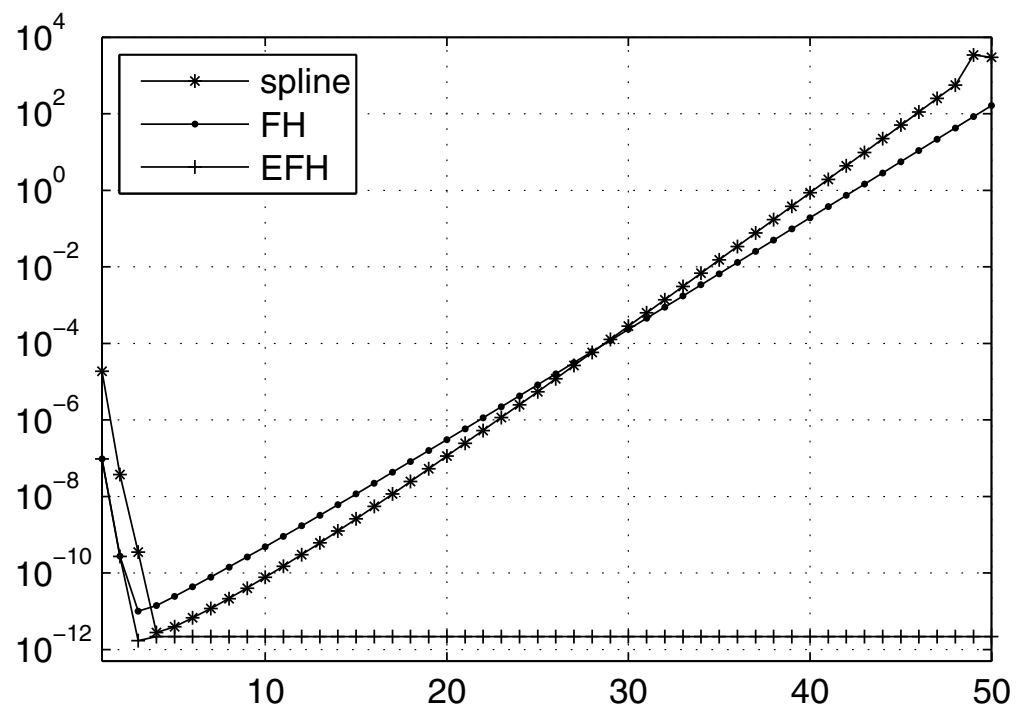

Figure 6. Error behaviour of spline, Floater-Hormann (FH) and extended Floater-Hormann (EFH) interpolation of $f_{1}$ with sign alternating $10^{-12}$-perturbation of the data, $n=1000$ and $1 \leq d \leq$ 50. 

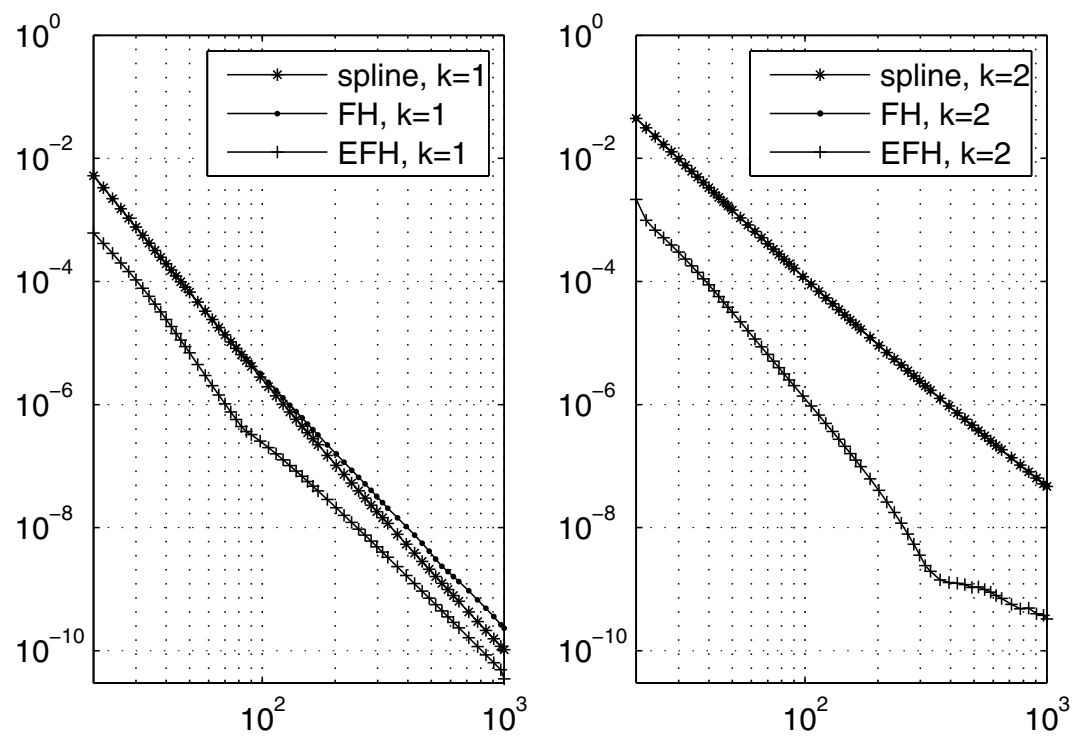

FiguRE 7. Error behaviour of spline, Floater-Hormann (FH) and extended Floater-Hormann (EFH) approximations of the first (left) and second derivative (right) of $f_{2}$ in $[-5,5]$ with $d=4$ and $20 \leq n \leq 1000$.

$10^{-12}$ is alternatively added and subtracted to the given data, i.e., to the sample of $f_{1}$ at equispaced points. In Figure 6 the error behaviour of the investigated interpolants of the perturbed $f_{1}$ in $[-5,5]$ with $n=1000$ is shown as a function of $d$. As the value of the theoretical convergence order of the spline and FloaterHormann interpolants varies from 2 to 51, a minimum is attained in the error but the latter increases exponentially thereafter. Once it reaches its minimum, the error in the extended Floater-Hormann interpolant, in contrast, remains smallest possible, namely $10^{-12}$, which is the perturbation of the data. One may therefore conclude that choosing an inadequate value for $d$ is much less likely with the extended Floater-Hormann interpolants than with the original family. Even more extreme examples confirm this observation: Floater-Hormann interpolation with $d$ too large and for severely perturbed data yields large deviations towards the ends of the interval, whereas the extended interpolants merely oscillate in the direct vicinity of the perturbation and with small amplitude. Every possible choice of $d$ becomes admissible with the extended family of rational interpolants: the interpolation of $\sin (x)$ in $[-5,5]$ with $n=50000$ and $d=200$ gives an error of $3 \cdot 10^{-12}$, this is in clear contrast to the error of 0.68 with the original Floater-Hormann interpolant.

We now turn our attention to the applications of the presented interpolants described in Section 4 The first and second derivatives of $f_{2}(x):=\sin (x)$ are approximated as suggested at the end of Section 4.1, namely by the rational interpolant with $d=4$ of the derivatives at the nodes of the interpolant of $f_{2}$, see (4.5) for the extended family and the analogous expression in [17] for the original family of rational interpolants. In Figure 7 where $k$ denotes the order of differentiation, 


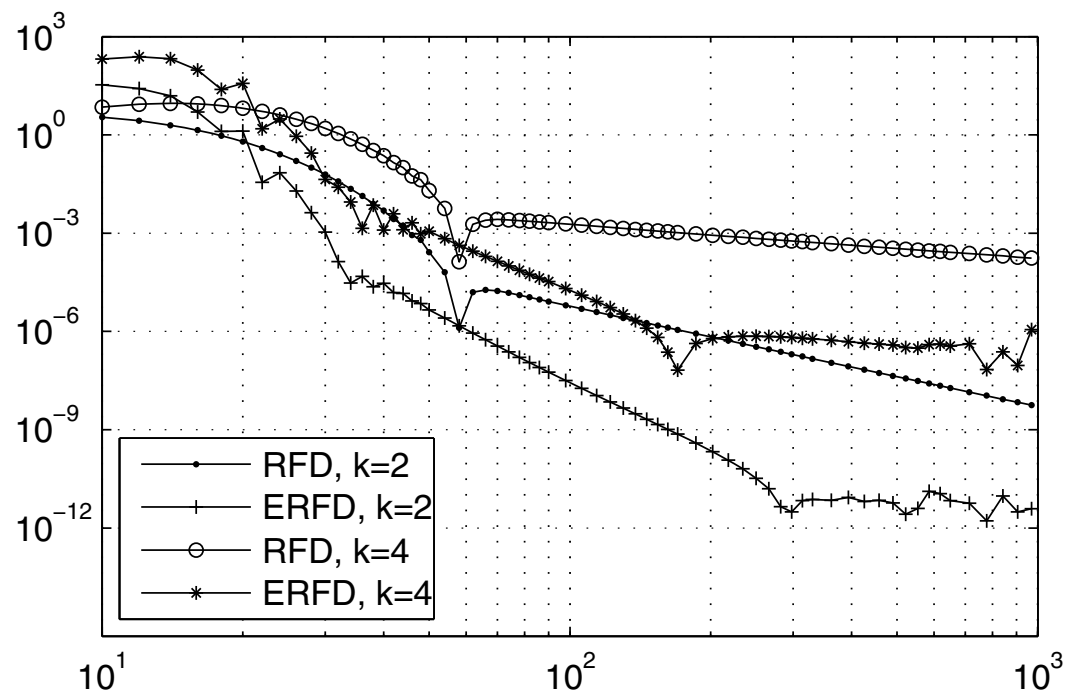

FIGURE 8. Error behaviour of one-sided RFD and ERFD (with $d=4$ and $10 \leq n \leq 1000$ ) approximations at $x=-5$ of the second and fourth derivatives of $f_{1}$ sampled in $[-5,5]$.

we see that the experimental convergence rates are similar with the three methods, as to be expected. The approximation based on the extended family yields smaller errors in both cases, with a remarkable difference in the approximation of the second derivative, where the errors with the methods based on the spline interpolant of order 5 , respectively, the original family, almost coincide.

One-sided rational finite difference approximation at the ends and RFD approximation near the ends of the interval are very successful for large numbers of nodes, as already noticed in [17]. One-sided ERFD approximation at $x=-5$ with $d=4$ of the second and fourth derivatives of $f_{1}$, sampled in $[-5,5]$, still improves upon RFD; see Figure 8. In this example the experimental rates of convergence are even larger with the ERFD method; classical FD approximation fails, mainly because of Runge's phenomenon [17. The fact that the extended family of rational interpolants displays reduced oscillations towards the ends of the interval as compared to the original family definitely helps in this application, in addition to the better conditioning.

Finally we consider the approximation of antiderivatives and integrals of $f_{3}(x):=$ $\sin (100 x)+100$ in the interval $[0,1]$ using rational interpolants with $d=5$ (the function $f_{3}$ is chosen so as to avoid the approximation of 0 while integrating $\sin (100 x)$ over one period). The errors in the indirect rational methods for the approximation of an antiderivative are larger than those obtained with the antiderivative of the spline interpolant of order 6; see Figure 9. This result was to be expected since the former methods use the differentiation matrix for the computation of the derivative at the nodes of the rational interpolant approximating the antiderivative, which does not improve the convergence rates; numerical experiments with the IRQ in [16] revealed experimental orders $O\left(h^{d+1 / 2}\right)$. It must, nevertheless, be kept in mind that the indirect rational methods give analytic approximations of antiderivatives. 


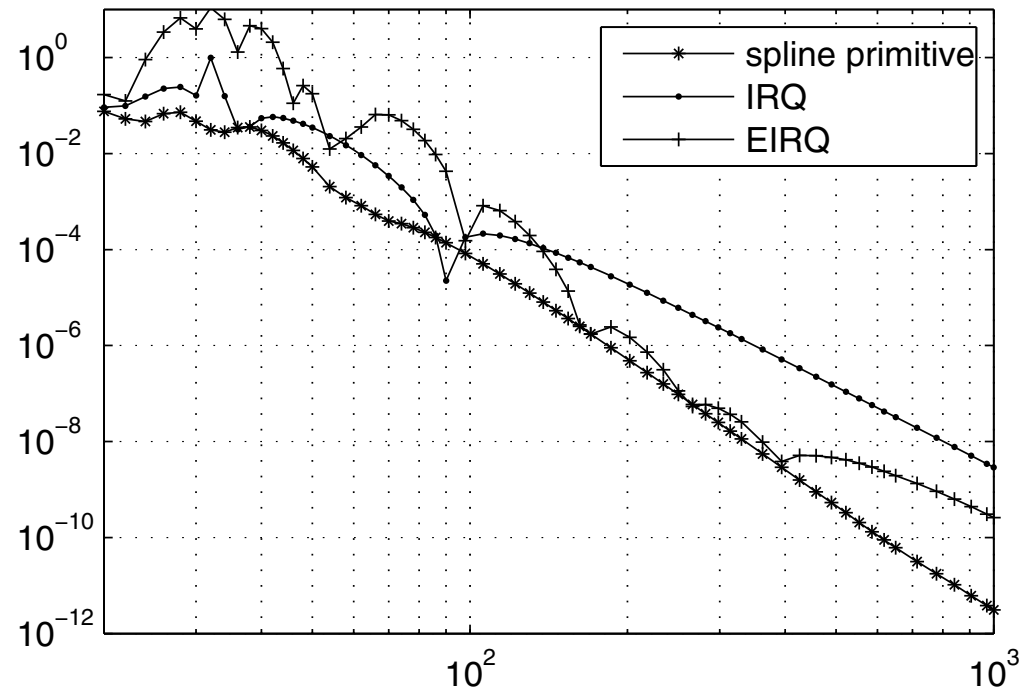

FIgURE 9. Error behaviour of spline antiderivative, IRQ and EIRQ of $f_{3}$ in $[0,1]$ with $d=5$ and $20 \leq n \leq 1000$.

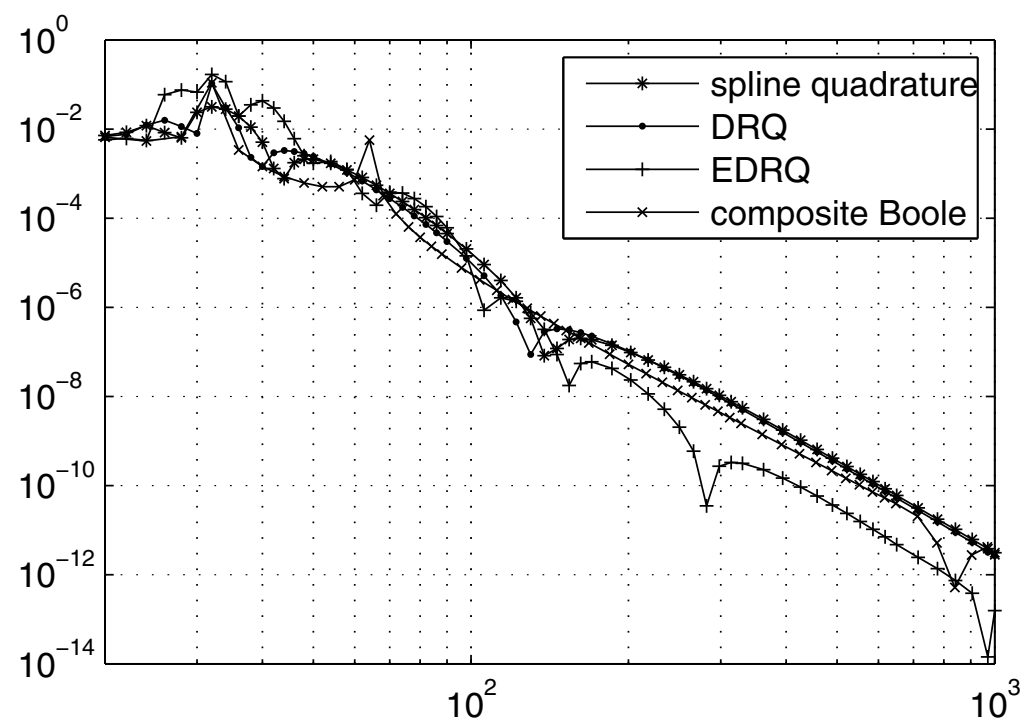

FiguRE 10. Error behaviour of spline quadrature, DRQ, EDRQ and Boole's rule for the integral of $f_{3}$ in $[0,1]$ with $d=5$ and $20 \leq n \leq 1000$.

Figure 10 displays the errors in the approximation of the integral of $f_{3}$ over the interval $[0,1]$ by the integral of the spline interpolant of order 6 of $f_{3}$, by DRQ 
and EDRQ with $d=5$ as well as the composite Boole rule, which is the composite Newton-Cotes rule of order 6 . To be specific about the direct quadrature rules, we precise that the integrals of the rational interpolants are approximated here by the 1000-points Gauss-Legendre rule computed with the Chebfun [25] command legpts. For small values of $n$, the four methods yield similar results. With larger values of $n(\geq 150)$, the error curves show smallest errors in the EDRQ. In this example EDRQ beats Boole's rule, whereas DRQ does not. Numerical experiments reveal that there are even fewer negative quadrature weights in the EDRQ rules than in the DRQ ones, which already contain only few of them for small values of $d[16$.

\section{Conclusion}

In this work, we have extended the family of Floater-Hormann barycentric rational interpolants for analytic and well-conditioned interpolation at equispaced points. The theoretical convergence rates of the interpolation and the methods for the approximation of derivatives, integrals and antiderivatives derived from the rational functions are similar to those obtained with the original Floater-Hormann family. However, the condition of the new interpolation scheme, as measured by its sensitivity to perturbations, is much more favourable and the parameter $d$ may be chosen significantly larger than with the original family. More generally, numerical examples show that the absolute errors in the new methods tend to be smaller, especially for large numbers of interpolation nodes.

\section{ACKNOWLEDGEMENT}

The author expresses his thankfulness to Jean-Paul Berrut and Nick Trefethen for discussing draft versions of the present paper.

\section{REFERENCES}

[1] B. K. Alpert, High-order quadratures for integral operators with singular kernels, J. Comput. Math. Appl. 60 (1995), 367-378. MR1357317 (96h:65037)

[2] R. Baltensperger and J.-P. Berrut, The errors in calculating the pseudospectral differentiation matrices for Čebyšev-Gauss-Lobatto points, Comput. Math. Appl. 37 (1999), 41-48, Corrigenda in Comput. Math. Appl. 38 (1999), 119. MR1664260 (99h:65173)

[3] R. Baltensperger, J.-P. Berrut, and B. Noël, Exponential convergence of a linear rational interpolant between transformed Chebyshev points, Math. Comp. 68 (1999), 1109-1120. MR.1642809 (99k:41016)

[4] J.-P. Berrut, Rational functions for guaranteed and experimentally well-conditioned global interpolation, Comput. Math. Appl. 15 (1988), 1-16. MR.937561 (89b:65029)

[5] J.-P. Berrut and R. Baltensperger, The linear rational pseudospectral method for boundary value problems, BIT 41 (2001), 868-879. MR2058847 (2005d:65124)

[6] J.-P. Berrut, M. S. Floater, and G. Klein, Convergence rates of derivatives of a family of barycentric rational interpolants, Appl. Numer. Math. 61 (2011), 989-1000. MR2806141

[7] J.-P. Berrut and H. D. Mittelmann, Lebesgue constant minimizing linear rational interpolation of continuous functions over the interval, Comput. Math. Appl. 33 (1997), 77-86. MR.1449215 (98c:41015)

[8] J.-P. Berrut and L. N. Trefethen, Barycentric Lagrange interpolation, SIAM Rev. 46 (2004), 501-517. MR2115059 (2005k:65018)

[9] L. Bos, S. De Marchi, and K. Hormann, On the Lebesgue constant of Berrut's rational interpolant at equidistant nodes, J. Comput. Appl. Math. 236 (2011), 504-510. MR2843034

[10] L. Bos, S. De Marchi, K. Hormann, and G. Klein, On the Lebesgue constant of barycentric rational interpolation at equidistant nodes, Numer. Math. 121 (2012), 461-471. MR2929075 
[11] L. Brutman, On the Lebesgue function for polynomial interpolation, SIAM J. Numer. Anal. 15 (1978), 694-704. MR0510554 (58:23250)

[12] M. S. Floater and K. Hormann, Barycentric rational interpolation with no poles and high rates of approximation, Numer. Math. 107 (2007), 315-331. MR2328849 (2008f:41006)

[13] B. Fornberg, A Practical Guide to Pseudospectral Methods, Cambridge University Press, Cambridge, 1996. MR1386891 (97g:65001)

[14] K. Hormann, G. Klein, S. De Marchi, Barycentric rational interpolation at quasi-equidistant nodes, Dolomites Res. Notes Approx. 5 (2012), 1-6.

[15] D. Huybrechs, On the Fourier extension of nonperiodic functions, SIAM J. Numer. Anal. 47 (2010), 4326-4355. MR2585189(2010m:42004)

[16] G. Klein and J.-P. Berrut, Linear barycentric rational quadrature, BIT 52 (2012), 407-424. MR2931356

[17] _ Linear rational finite differences from derivatives of barycentric rational interpolants, SIAM J. Numer. Anal. 50 (2012), 643-656. MR2914279

[18] R. B. Platte, L. N. Trefethen, and A. B. J. Kuijlaars, Impossibility of fast stable approximation of analytic functions from equispaced samples, SIAM Rev. 53 (2011), 308-318. MR2806639

[19] M. J. D. Powell, Approximation Theory and Methods, Cambridge University Press, Cambridge, 1981. MR604014(82f:41001)

[20] C. Schneider and W. Werner, Some new aspects of rational interpolation, Math. Comp. 47 (1986), 285-299. MR842136 (87k:65012)

[21] A. Schönhage, Fehlerfortpflanzung bei Interpolation, Numer. Math. 3 (1961), 62-71. MR0119389(22:10153)

[22] G. D. Smith, Numerical Solution of Partial Differential Equations: Finite Difference Methods, 3rd ed., Clarendon Press, Oxford, 1985. MR827497(87c:65002)

[23] T. W. Tee, An Adaptive Rational Spectral Method for Differential Equations with Rapidly Varying Solutions, PhD thesis, University of Oxford, 2006.

[24] T. W. Tee and L. N. Trefethen, A rational spectral collocation method with adaptively transformed Chebyshev grid points, SIAM J. Sci. Comp., 28 (2006), 1798-1811. MR2272189 (2008k:65213)

[25] L. N. Trefethen and others, Chebfun Version 4.0, The Chebfun Development Team, 2011, http://www.maths.ox.ac.uk/chebfun/.

[26] L. N. Trefethen and J. A. C. Weideman, Two results on polynomial interpolation in equally spaced points, J. Approx. Theory 65 (1991), 247-260. MR.1109406 (92e:41007)

Department of Mathematics, University of Fribourg, Perolles, CH-1700 Fribourg, SWITZERLAND

E-mail address: georges.klein@unifr.ch 